\title{
Merging Remote Laboratories and Enquiry-based Learning for STEM Education
}

\author{
http://dx.doi.org/10.3991/ijoe.v10i6.3997 \\ Ananda Maiti, Andrew D. Maxwell, Alexander A. Kist and Lindy Orwin \\ University of Southern Queensland, Toowoomba, Australia
}

\begin{abstract}
Practical learning activities are effective ways to increase the interest of students in Science, Technology, Engineering and Mathematics (STEM) topics. Attracting school students to STEM careers is a challenge in many parts of the world. Remote Access Laboratories (RAL) allow for offsite control of state-of-the-art science and technology experiments. Such learning activities, while mostly used in universities, can also provide additional learning opportunities in schools. Current RAL technologies are targeted at undergraduate engineering education and offer limited operational autonomy. Opportunities for collaboration and experiment design are not well supported. However, both are important components of STEM education in schools. This paper discusses how RAL can be adapted to better support enquiry based learning in the context of STEM education. The limitations of current architectures are discussed and approaches to address these are presented.
\end{abstract}

Index Terms - remote laboratories; instructional design; elearning; enquiry-based learning; STEM

\section{INTRODUCTION}

Science, Technology, Engineering and Mathematics (STEM) are key subjects in school education that develop skills required to progress into the science and technology related tertiary study and careers. Enquiry-based learning (EBL), as well as problem-based and project-based learning, are effective ways to teach STEM in school education[1]. These teaching strategies encourage students to think on their own, work in teams, design solutions and study their effects to gain knowledge and experience of STEM concepts. Generally, these strategies are limited to the local environment at schools. Collaborations between schools to share activities and use them remotely could provide a number of benefits

Remote Access Laboratories (RAL) are online interactive multimedia platforms that allow students to access and control devices and experiments at distant locations remotely. The student can get experiment result data as well as audio/video feedback of the remote device. RAL has been used extensively in universities worldwide [2]. RAL may be used to aid in this goal of teaching STEM with EBL providing access for more students to a more diverse range of experiments and creating the opportunity for collaborative networks of students who are using these experiments to share, compare and aggregate data.

Previous research has shown that current RAL systems are deficient in features to support STEM education [2]. Most of the RAL are initiated to complement the regular laboratory teaching at universities as a means to increase accessibility to increasing number of enrolled students.
Hence activities are designed to be services provided by universities and have a fixed set of experiments that are directly related to the university curriculum. The experiments are usually pre-configured and students have to collect data by changing experimental conditions. Most of these RAL allow little collaboration between students.

In this paper, Remote Access Laboratory technology is merged with enquiry-based learning methodologies to create an integrated architecture that can support STEM Education more efficiently than either of these individually. The limitations of current RAL systems for using it in enquiry-based learning in STEM education are analyzed. A distributed RAL architecture is proposed and initial trial results are discussed. A system model for RAL is presented and used to determine the similarity between the RAL and on-site laboratories and determine the areas to expand.

In Section II the current approaches and results of introducing RAL experiments into primary and secondary schools, RAL system evaluation models and the status of Learning Management Systems (LMS) used to address pedagogical issues in the use of RAL in engineering education are discussed. In Section III RAL is defined in two dimensions and expected characteristics of RAL in STEM education with EBL is described. Section IV proposes the distributed model of RAL systems and the ways to extend RAL features followed by analysis of limitations of the LMS and other ways to implement the proposed system in Section V.

\section{RELATED WORKS}

\section{A. RAL in K12 education}

Recently a number of studies have been conducted on the impact of RAL in learning of K12 children by offering experiments from university to schools.

At a primary school in Slovakia in 2011/2012 one experiment from a university was made available to students to study the effects of project-based learning in a RAL environment on their learning development [3]. This experiment was a sensor-type experiment which collected weather data from remote stations. Several project-based learning activities were included in the test that asked students aged 12-13 to collect data, analyze them and identify conclusive results on the observations. The results indicated that students the RAL environment performed better than students with didactic test. Remote experiments were regarded as interesting and a project based approach was rated as good.

Similar studies for secondary schools were carried out in Australia withe the Labshare RAL [4]. This study also introduced specialized hardware implemented at the uni- 
versity for teachers and students in a secondary school. The students, mostly for year 9-11, were given access to interactive experiments in physics and given a set of experimental activities to conduct. The advantages of RAL stated by majority of the students were the ease of learning new things and ease of gathering data. However, most students and teachers regarded that there was serious lack in team work or collaboration which is an essential part of laboratory learning. Over 50\% students stated that they would have preferred to operate the equipment themselves in their own way rather than a 'ready-to-go' experiment.

Another application of RAL systems in schools has been reported by [5]. Students from two countries, Australia and Japan, were involved in a collaborative exercise where they shared design plans and an activity created by the other groups. Each group first constructed their own local activity. Then one group created a setup based on the findings of the second group which then ran the activity remotely. The entire activity was done under the supervision of researchers, but the experiment setups were created by the school students. The greater autonomy in this case allowed more interaction between students and motivated them.

It may be noted that in the first two cases, the RAL experiments were provided as static readymade experiment. This does not help with improving design skills of the students and makes it very easy to collect data virtually without any scope of error. This does not give the students an authentic experience about the different pitfalls of setting up a rig. Also, since there was no design phase, there was very little collaboration between students during the use of the experiment. For STEM education both design and collaboration are important. In the third case, students were asked to design their own setup which, although supervised, suggests that young learners are capable of designing their own experiments.

\section{B. Existing RAL Pedagogical Models}

To understand the STEM requirements of RAL systems, an educational model must be used. A comprehensive comparison between the structure and expected learning outcomes of hands-on and remote laboratory has been done before [6]. A 4-dimensional model of evaluating a laboratory was suggested. It concluded that compared to on-site laboratories, RAL are similar in two of the four dimensions - developing professional skills and conceptual understanding, a little short in the third - social skills and very poor in case of the fourth - design skills. Another work used university- based remote experiments to teach physics education in primary schools [3]. This work concluded that there is a relation between students' learning and active participation in an experiment. It used a 3dimensional model by removing the design skills.

\section{Current use of LMS in RAL}

To manage the learning aspects of RAL a Learning Management System (LMS) or Content Management system (CMS) is often used [7]. The LMS are essentially responsible for authentication, user tracking and scheduling in the RLMS. LMS and the RAL follow e-learning standards like SCORM (Sharable Content Object Reference Model), IMS or QTI and uses LMS APIs for intercommunications [8]. LMS from different RAL systems have been integrated to share resources between them [9]. The LMS contains experiment documentation, collabora- tive tools, the command interface and audio/video feed. The Lila Project [10] created a portal that integrates RAL systems from various universities where experiments are available in form of SCORM [11] entities. This way the RLMS meets the pedagogical needs of laboratory education equivalent to that of the on-site laboratories.

\section{THE RAL EDUCATIONAL MOdEL}

To understand the current state of RAL capabilities and to identify which additions are required, an educational system model of RAL in general is presented in this section. RAL systems may be described by analyzing the two most basic dimensions based on the above $[3,6]$ : operational autonomy and pedagogy. Operational autonomy is the scale of technical flexibility offered to the student in an experimental activity. It is low when the students have access to only a fixed experiment rig experiment that needs minimal (or no) interaction to get the data; and high when the students can create and alter the experiment conditions to get different results. Pedagogy is the conceptual learning values associated to an experiment i.e. how the experiment is presented and done by the student like enquiry-based learning and project-based learning. A static pedagogy indicates that the RAL experiment replicates the most essential components of learning from the corresponding hands-on experiment and more flexibility in pedagogy implies that RAL experiments are presented in innovative ways taking advantage of ICT for delivery, motivation, flexibility and student engagement.

Figure 1 depicts four quadrants that indicate different levels of operational autonomy and pedagogy. Both of these must go hand in hand and with the increased complexity in pedagogical needs, the complexity and requirements standards of operational autonomy also increase.

\section{A. Exploring the System Model of RAL}

Current RAL systems have less flexibility in operational autonomy and associated pedagogy [12]. These are suitable for development of general concepts in higher education where equipment used is expensive. Experiments are often measurements generating huge amount of data from some phenomenon within certain conditions.

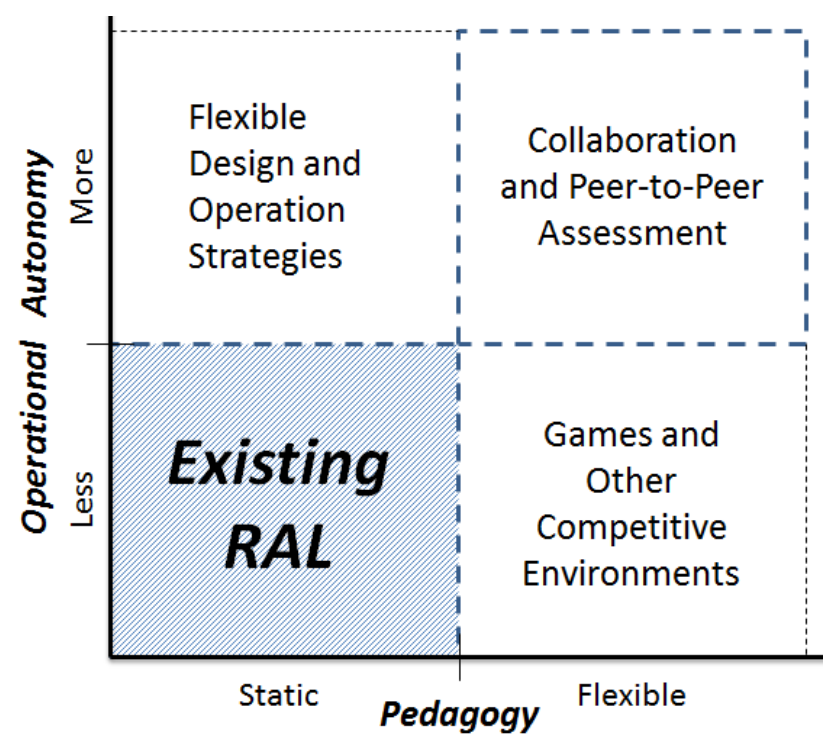

Figure 1. The RAL Extension 
Experiments do not need to be customized according to specific problem sets for users to use them. Users are prohibited from designing rigs. However, in STEM, to understand a concept, one must build, run and see what happens with the experiment. Students may want to share their results with others to get feedback and get new ideas from different perspectives of the same problem. Pedagogical needs of RAL systems are now limited to what is available in a hands-on laboratory as shown in [6]. The concepts are understood as one would read, perform and understand them in an onsite laboratory.

\section{B. Nature of Experiments}

STEM experiments differ from higher education experiments. STEM experiments may be easily constructed but often creating the rig or setting up the experiment is an important part of the learning experience. This allows the students to better understand concepts and problems relating to the activity which is the main challenge. In higher education laboratories, on the other hand, the equipment is often expensive, proprietary and hard to reproduce. This also means that many experimental setups are static. Users of the experiments are not required to design or build the rigs that support the practical activities.

Enquiry-based learning [13] in STEM aims to make students think and find solutions to a problem by themselves. If this approach is applied in the context of RAL, experiments cannot be reduced to set of instruction. There is a need to present the activity as a problem. Solutions and approaches through which the outcomes are achieved may vary; however, the system has to be able to support students in implementing an experimental rig and related procedures.

\section{What should happen for STEM?}

The enquiry based learning methodologies encourage students to think of different solutions to a given problem on their own. Usually a given activity produces a question that needs to be answered in order to understand the activity. The cycle of enquiry based learning then follows as: investigation on the topic to find out more details, create a solution typically something physical, observe and record the outcomes and discuss the results among peers. The cycle goes on until the results are perfected to the hypothesis in the 'ask' or 'investigation' phase. In enquiry-based learning for STEM activities [14] the following are the most common steps performed by students:

- Given a problem, the first step is to formulate the problem statement that raises questions to the users.

- Prepare a hypothesis of the given problem i.e. what is ideal and most likely?

- Decide on subsequent required experimentations to test the hypothesis.

- Creating an interface that gives proper reflection of the experiment to be performed in a real environment.

- Take measurements and collect data from experiments and analyze them.

- Take cue from other users' results when required, for guidance.

- Teachers are able to facilitate and confirm the correctness of the result obtained.

The EBL stages can be combined with the distributed RAL as follows (see Figure 2):

1. In the investigation phase after students have gone through the concepts and are ready to make their own design, they can look up in the RAL systems about what others have done.

2. During the create phase they create their own setup to test the hypothesis and use them.

3. Then they use others' system and compare them to find the differences and understand the concepts and improve their own design.

4. Once the setup is finalized, the setup can be put on the RAL system for others to use.

Thus, the 'create' and 'discussion' phases of EBL can be easily incorporated into and improved by the distributed RAL system. Clearly, a client-server LMS and RAL cannot support these kinds of activities and thus both the dimensions of RAL must be extended.

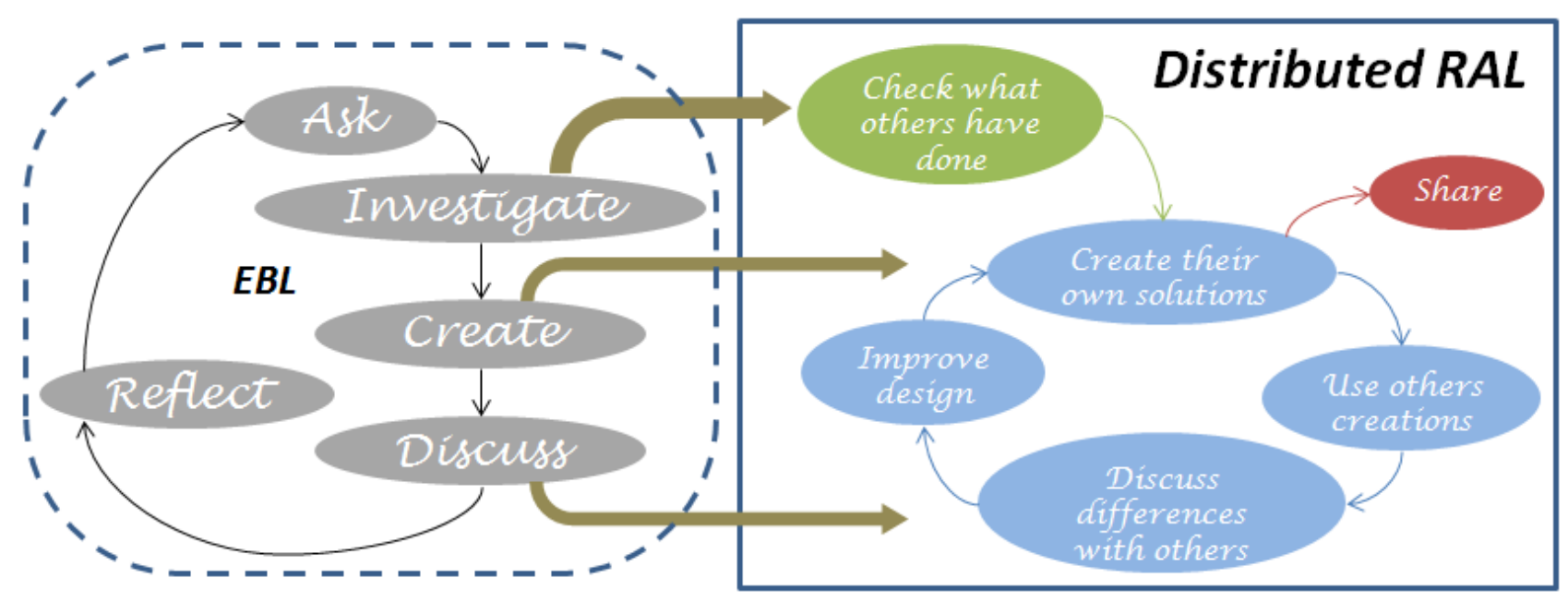

Figure 2. The phases of EBL for STEM (left side) extended to include the RAL features (right side) 


\section{EXAPANDING RAL}

A distributed RAL approach may be followed to extend the RAL system. In a distrusted approach, the students themselves become providers of the experiments and use each other's creations. The RAL system may be extended in the following ways:

\section{A. Increasing the scope of design and operation}

When increasing the design scope, a student must be allowed to plan and design their own setups for a given problem. Designing a STEM experiment will include the following steps:

i. Assemble an equipment setup.

ii. Program and run experiments locally.

iii. Share the experiment with other students by putting it on the Internet.

Students are exposed to several high end technologies from a young age. So they become capable in learning and using simple electronic devices ranging from programmable robotics like LEGO Mindstorms to mobile phones. These are consumer electronics that are available easily. To run an experiment requires a student to program the different parts of the rig so that they can communicate with each other and the internet. The only way to put a rig on the internet is by using a network enabled computing device. This raises the question of student's capability to program a rig.

With computers fast becoming an integral part of our lives, there are several graphical programming languages being taught to young students today such as Scratch from the MIT, Alice, Tynker and LEGO's LabVIEW based language. These programing languages are able to deliver the same capability of any high level language including multithreading, process communication and programming constructs. These languages may be easily used by students to create their own experiments. The entire scheme gives them full flexibility to think on the problem and come up with their own solutions.

\section{B. Flexibility in organization of experiments}

Another problem with the existing RAL infrastructure is the way the experiments are handled. From a user's point of view, the experiments offered are static to-do lists. This limits the RAL systems to provide the same capability as that of on-site laboratories. In a distributed form, the RAL experiment list is not static and users can upload whatever they want. As experiments are typically created following a curriculum, the nature of the experiments remains same but the way they are implemented differs from user to user. This provides a competitive or even collaborative environment and this platform may be used to develop a game-based RAL pedagogy [15] where students can achieve certain levels and milestones for successfully creating and completing their own and others' experiments. One manner of continuing activities and providing context is through a "Quest". This is a combined group of activities with a greater common goal, and may serve to create a learning path for a more abstract concept to be learnt.

\section{Quest-based Interface}

The decision to approach the user experience of RALfie as a quest-based game aims to capitalize on the motivation, engagement, communication and collaboration op- portunities afforded by the use of game elements. Creating an ongoing game-like interface has the potential to provide pedagogical and organizational opportunities to engage learners at cognitive, emotional and social levels. The design aims to create a sustainable, self-sufficient community with shared interests and distributed expertise.

Contextualizing the content in a story-based system of quests that incorporate the use or making of RAL binds the collection of disparate experiments into an ongoing, related story providing a context for informal learning. This does not prevent individual quests or groups of quests being used in formal education programs. Evidence shows that students do more work on average and achieve higher grades overall in a quest-based course when compared to traditional courses [16]. Quests and collections of related quests (quest chains) may involve the use of experiments or the making of RAL or some of both.

The reward and reputation system provides users with a system for tracking their own learning and the learning of others as well as linking together related quests. Its role is to stimulate participation and maintain engagement with STEM learning in a fun and challenging way. Players' experience points accumulate to earn achievement badges and clusters of badges may aggregate into super badges. The achievement system has paths for makers as well as users and learners can transition seamlessly between the two roles or stay exclusively within one role. A system of ratings is used to determine the popularity of quests as well as provide a feedback loop to the quest and experiment creators. During the prototyping stage, some of the administration of user generated content has been managed manually but a system for user submission and peer moderation will be a requirement in the final system.

To connect users, makers and enthusiasts and build an ongoing, self-sustaining, online community of STEM learners, RALfie's online community of peer learning support is modeled on gamer guilds. These online communities are designed around the social principles of constructivist learning and connectivism [17]. The "guilds" provide a mechanism for the wider community of STEM professionals, university students, academics and enthusiasts to contribute expertise to meet the needs of younger and less experienced learners. The system will capitalize on the affordances of a range of synchronous and asynchronous online communication technologies that currently includes discussion forums; calendars and rosters; polls, ratings and surveys; file sharing; media galleries for videos and images; and journals using blogs but could expand to explore roles for micro blogging; social media; virtual worlds; and voice and video chat using avatars. Guilds intend to provide a support community that addresses technical and maker needs and also fosters a positive attitude to STEM especially for ongoing users of the environment in informal learning contexts.

\section{Collaboration}

When both dimension of the RAL are increased, there is an added advantage of collaboration between students as peers. As students are running each other's experiments, they are capable of providing feedback on their peer's experiments and learning from each other. When experiments are designed and run collaboratively, these add to the learning outcomes $[18,19]$. Students can also help each other by 'Reciprocal Teaching' as stated in [19] i.e. each student upon completing an activity contributes 
PAPER

their experiment and knowledge to the system which is then used by other new students in the system. 3D virtual world technologies have been used successfully used to provide a hands-on technology to students via distance collaboration platform [20] and can be used here in a similar manner.

\section{SYSTEM FEASIBILITY}

The RLMS needs to be considerably modified and the features extended to accomodate the needs of the propsed RAL architecture with Enquiry-Based Learning (EBL). The feasibitly of such is discussed here with respect to the learning management, physical networking and control capabilties of the RAL.

\section{A. LMS features to support RAL for STEM}

The LMS-RAL integration has been designed with several key pedagogical factors in mind especially collaboration. Team-work is an important part of RAL activities. It promotes communication skills and helps the group by bringing in different perspectives of the problem. There have been reports of LMS where such collaboration is possible [21-23]. Students can join their virtual groups in the LMS and start a joint session during which they interact with the equipment in turns and interact between themselves [24]. The LMS also provides a search and booking mechanism where students can find the experiments, associated data and book time for experiments.

However, for the proposed expansions, students need to create their own rigs, program them and create a user interface. These features are absent in the LMS. Also a game-based learning approach where students can learn in gradual steps and in a competitive environment is not available within the LMS yet.

\section{B. Network Support}

The distributed approach to RAL design requires a corresponding underlying Network Architecture that enables direct end to end or student to student access. Cloud computing is a distributed approach of communication and control of data in information systems. It is the basis of many large industries today and has well-established standards. Peer-to-Peer networking systems are a feature of the cloud computing services where two anonymous users can connect and share data without the need of any centralized server system e.g. Skype.

The storage of and access to data regarding the experiments are in two phases (see Figure 3):

1. Experiment management: In case of RAL, the data about the experiments including experiment list, documentation and gaming parameters and their relationship based on the gamification can be stored as either a central or global repository. The game- based logic of incentive related to badges and experience point (rewards and reputation) from an experiment as well as association with dedicated communities is stored at this layer.

2. Experiment discovery and access: Despite the storage mechanism of the data related to the experiment, the actual hardware hosted by users can be directly reached by other users for actual use of their experiment's rigs.

The Web Services (WS) [25] methodology has been used in traditional RAL systems. A fixed set of web services are created and associated with the individual components or functions of the instrument. The experiment is run by calling the respective WS. The WS may be stored in the Centralized storage mechanism e.g. UDDI along with the game logics. This approach is however slow because it initiates HTTP like connections procedure each time a WS function is called when it is required to be executed. It is considerably complex to setup such functions in the object-oriented programming, particularly by young students and school teachers. These systems are very rigid and static architecture that are difficult to alter swiftly and be adaptive to network usage conditions.

The alternate to the WS is the overlay network or VPN solutions. These network architectures let the individual end user nodes in the system to be connected to each other without any central authorization system. These are a logical network built on top of a physical network. Such system are easy to deploy and can have customized interface and protocols for communication and visualizing the experiments data and results. These systems are flexible and dynamic and thus more fault-tolerant.

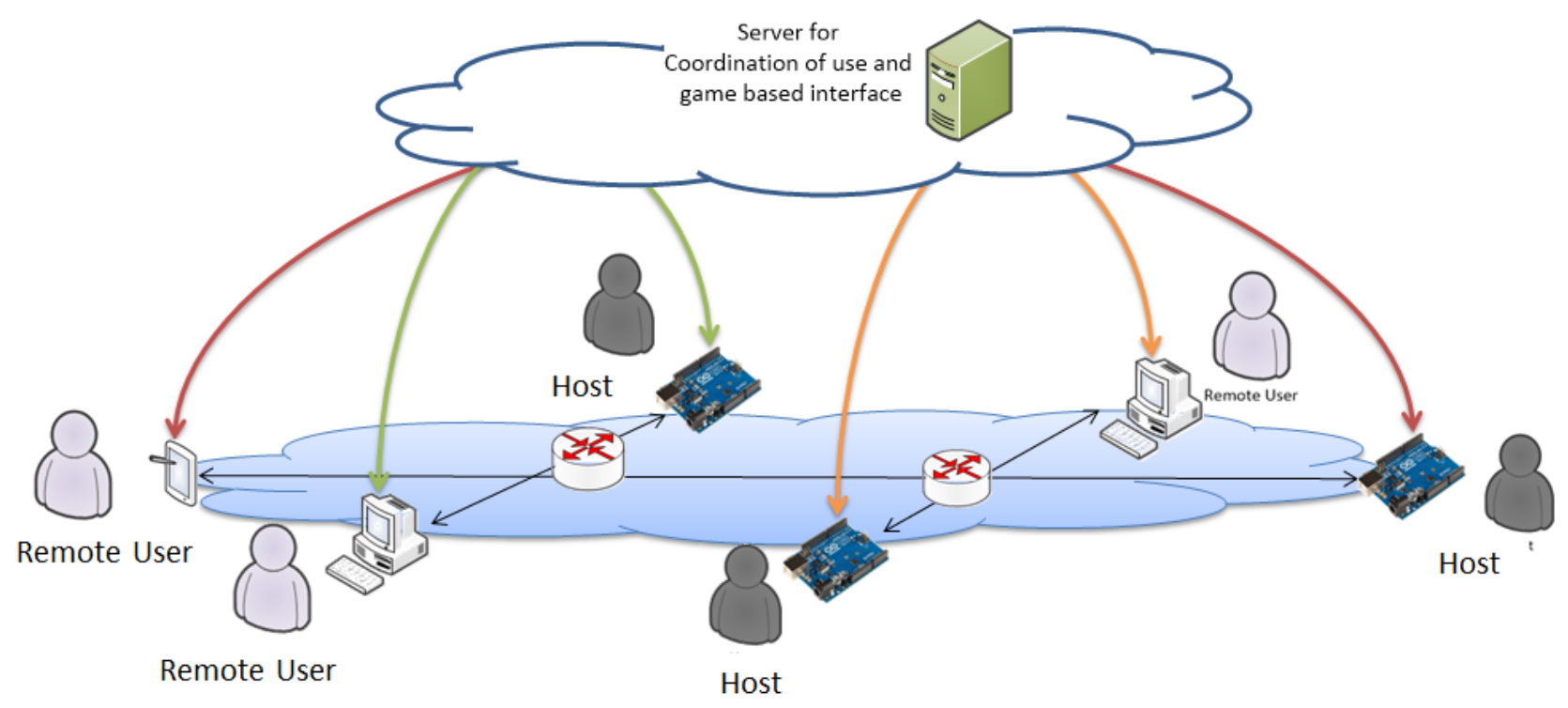

Figure 3. The distributed RAL architecture 


\section{Distributed Control}

In terms of feasibility of using and/or creating an experimental rig, several options exist.

- Use of existing equipment designed for and installed as part of traditional RAL systems. Translation services would then bridge between systems;

- Use of existing equipment designed for desktop laboratory support;

- Use of customized laboratory equipment;

Whilst there are variations of the above, each of these typically will involve expensive equipment not typically available to all users. The counterbalance for this is the lost cost activity. This introduces a "Maker" element to the project where activities and experimental apparatus are designed to be easily constructed and replicated. For instance:

- Pre-assembled kit of open source or easily obtained hardware and software (LEGO TM, Meccano TM, Arduino $^{\mathrm{TM}}$, BeableBone ${ }^{\mathrm{TM}}$ )

- Pre-packaged collection of equipment, with instructions

- Instructions provided with links to manufacturers' list of parts

- Instructions provided utilizing common construction elements (low cost and readily found, found in the "parts" box of many laboratories or enthusiast's workshops, through to elements of e-wast, and discarded equipment and computers.

Consideration is also required regarding the reliability of equipment. If lower cost equipment is utilized, reliability can often be lowered. Low cost equipment however is easy to replicate either on the same-site, or distributed via P2P networks. This offers a unique advantage by allowing more participants to create and host activities thus providing for redundancy in the system.

The level of flexibility required for creating a STEM activity is practically available to average users $[5,26]$. However, the creation and hosting process have to be standardized.

\section{Pedagogical FEASIBILITy}

In the last section it is clear that the technology and tools required to establish a P2P RAL is feasibly available. This section describes the trials and their analysis with students.

\section{A. Trial with Students}

RALfie (Remote Access Laboratories for Fun, Innovation and Education) is being developed with the aim of creating a distributed maker-based RAL system with the proposed extended RAL features. A series of trials have been developed to allow investigation into both technical competence of the target audience and to inform aspects of how best to deliver peer-to-peer technical services. Participants training and education needs, as well as teacher professional development needs of teachers, are also being investigated.

A trial was held with 15 school students aged between 6 and 17 yrs. The trial was used to establish the feasibility of a distributed system and its requirements. The aim was to find out if the student can understand, create and test a rig and work on the concept of sharing their creations with others. This trial was to answer specific questions regarding pre-existing and trial-developed conceptual understanding by children of the RALfie concepts and how to construct and configure a peer-to-peer remote access laboratory.

In order to provide a context, three initial activities were provided to the students.

- 'Mouse in the house' - a control activity designed to explore problem based learning within the RALfie environment (Activity 1).

- 'All the Way Home' - a programming activity to highlight the difficulty of controlling a potentially unstable activity remotely (Activity 2).

- 'Shrimp Ahoy' - a static activity where the primary objective is observation, and the creation of an 'activity' around the provided technology (Activity 3 ).

Each of these activities was designed to explore a different aspect of EBL, and designed to include a wide range of background experiences, technical competencies, and interests.

Technically, each activity was based on a different implementation process. Activity 1 was based on a 2 axis gimbal mount and was created with LEGO Mindstorms ${ }^{\mathrm{TM}}$, and controlled through a remote desktop protocol (RDP). The gimbal mount was purposely ill-designed with a mechanical issue that would sometimes reverse the controls to further increase the likelihood of problem solving occurring by users. The goal of the activity was to mobilize two white marbles (the "mice") on top of a flat rolling plate, into a containment area (the "house") using 2 axes of freedom, and remote cameras for observation.

Activity 2 involved programming an entirely untethered mobile robot (built from LEGO Mindstorms ${ }^{\mathrm{TM}}$ ) after establishing a Bluetooth link, to mobiles around a "line follower" track. The robot was unconstrained by barriers, or electrical tether, and thus increased the likelihood and consequences of the program not working, or control being lost (where the robot would fall off the table). The establishment of serious and real consequences for incorrect operation was designed to increase discussion amongst team members around safe control and placement of web-cameras for observation.

Activity 3 was designed as only an observational activity with a minor component of control. An aquarium containing a school of 15 feeder-shrimp, multiple webcameras, and remotely controlled lighting was provided. The purpose of the activity was to examine the shrimp's activity over time as a "school", as well as their predisposition to move away/towards light combinations.

\section{B. Prelimenary Trial Results}

The trial event was conducted over a 4 hour time period. Data collected included interviews and focus group discussions with the children during and post-event, as well as conceptual drawings of key RALfie concepts. In addition to this, 5 video cameras were used to capture discussions, as well as in-room interactions with the equipment and between participants. All activities used a Think-Aloud Protocol to capture participants thinking and internal cognition. This data was initially screened through a thematic analysis to highlight specific recurring 
themes and understandings relating to activities at the time, as well as analysis of specific questions asked.

\section{1) EBL Processes}

Previously the iterative EBL workflow structure was presented: Ask-Investigate-Create-Discuss-Reflect. During this event each team and most participants moved through this workflow. Participants were provided with initial briefing information and then able to ask questions regarding the systems configuration and the activity. Participants were then able to investigate the inner workings of each activity and identify issues and problems. Initially the "create" stage was confined to connecting equipment in a pre-set manner. Discussion and reflection occurred as each team used the activity locally and then remotely from another room. This served to increase discussion about potential issues with the initial activity's design.

In later stages of the trial, many participants chose to re-investigate the configuration of equipment we provided and extend its functionality. This demonstrated that where participants were "Makers" of activities, they were able to continue through the EBL workflow and achieve learning outcomes beyond the original design intent.

\section{2) Flexible Design and Operational Strategies}

In comparison with traditional RAL activities, it was immediately observed that participants who were unhappy with the configuration of activities and cameras as provided were able to modify, retrofit, and extend the activity beyond the original design. The use of less technology and ubiquitous construction methods (LEGO, Web-Cameras) contributed to the familiarity of the technology.

\section{3) Collaboration and Peer-to-Peer assessment}

No game or online environment was used in this particular trial; however opportunity for verbal communication was encouraged through a "thinking aloud" protocol. Teams were identified as self-organizing where knowledge was shared with participants during the trial, as might occur with online forums in a similar context. This served to provide a degree of mentoring of participants and sharing of expertise.

\section{4) Games and Other Competitive Environments}

Although a game system was not utilized during the trial, some activities did appeal to the competitive nature of the participants. This was observed as significantly increasing engagement, however not to the point where participants unable to complete the challenge were ostracized. This is most likely due to the technical nature of the activities, where this trial focused more on the systems rather than the activity.

\section{CONCLUSION}

This paper presents a new manner of looking at the creation and maintenance of Remote Access Laboratories (RAL). This is done by moving away from the more traditional centralized hosted method towards peer-to-peer methods. This has the potential to allow a wide community to access Science, Technology, Engineering and Mathematics (STEM) interdisciplinary learning. In this move, educational and support frameworks must be adapted to cater for less teacher-centric approaches, where both responsibility and opportunity for distributed learning lies with all participants. For this purpose, a system model of RAL is presented that provides an insight into what are the basic components of RAL and their relationship and ways to improve them through operational autonomy and constructionist pedagogy. This strategy is then used to extend the RAL features to support and Enquiry-based Learning strategies in STEM education with increased scope of design activities. By allowing participants to also make activities, a more diverse range of competencies can also be targeted in the technology areas of computer networking, computer science and programming.

\section{REFERENCES}

[1] A. Zollman, "Learning for STEM Literacy: STEM Literacy for Learning," School Science and Mathematics, vol. 112, pp. 12-19, 2012. http://dx.doi.org/10.1111/j.1949-8594.2012.00101.x

[2] A. Maiti, A. D. Maxwell and A. A. Kist, "An Overview of System Architectures for Remote Laboratories," in TALE 2013, pp. 661666, 2013.

[3] M. Kostelníková, M. Ožvoldová, "Remote Experiments in Primary School Science Education," International Journal of Online Engineering (iJOE), vol. 9(5), pp. 39-44, 2013.

[4] D. Lowe, P. Newcombe, and B. Stumpers, "Evaluation of the Use of Remote Laboratories for Secondary School Science Education," Research in Science Education, vol. 43, pp. 1197-1219, 2013/06/01 2013.

[5] A. Maxwell, R. Fogarty, P. Gibbings, K. Noble, A. A. Kist and W. Midgley, "Robot RAL-ly International - Promoting STEM in elementary school across international boundaries using remote access technology," in REV 2013, 2013, pp. 1-5.

[6] J. Ma and J. V. Nickerson, "Hands-on, simulated, and remote laboratories: A comparative literature review," ACM Comput. Surv., vol. 38, p. 7, 2006. http://dx.doi.org/10.1145/11 $\underline{32960.1132961}$

[7] M. Tawfik, E. S. Cristobal, A. Pesquera, R. Gil, S. Martin, G. Diaz, et al., "Shareable educational architectures for remote laboratories," in Technologies Applied to Electronics Teaching (TAEE), 2012, 2012, pp. 122-127.

[8] E. Sancristobal, R. G. Sergio Martín, C. Martínez, E. López, N. Oliva, F. Mur, et al., "Development and Interaction between LMS Services and Remote Labs," International Journal of Online Engineering (iJOE), vol. 4, p.35-41, 2008.

[9] E. Sancristobal, J. Garcia-Zubia, P. Orduna, M. Castro, M. Emaldi, and D. Lopez-de-Ipina, "Modelling remote laboratories integrations in e-learning tools through remote laboratories federation protocols," presented at the Proceedings of the 2012 IEEE Frontiers in Education Conference (FIE), 2012.

[10] A. Gallardo, T. Richter, P. Debicki, L. Bellido, V. Mateos, and V. Villagra, "A rig booking system for on-line laboratories," in Global Engineering Education Conference (EDUCON), 2011 IEEE, 2011, pp. 643-648.

[11] SCORM, Retrieved Nov 1, 2013, from: http://scorm.com/.

[12] A. Maiti, A.D. Maxwell and A. A. Kist, "Features, Trends and Characteristics of Remote Access Laboratory Management Systems", International Journal of Online Engineering (iJOE) 10 (2), 2014, pp. 30-37. http://dx.doi.org/10.3991/ijoe.v10i2.3221

[13] D. C. Edelson, D. N. Gordin , R. D. Pea, "Addressing the Challenges of Inquiry-Based Learning through Technology and Curriculum Design", The Journal of the Learning Sciences, vol. 8, pp. 391-450, 1999. http://dx.doi.org/10.1080/10508406.1999.9672075

[14] R. F. Owens, J. L. Hester, and W. H. Teale, "Where do you want to go today? Inquiry-based learning and technology integration," Reading Teacher, vol. 55, pp. 616-625, 2002.

[15] S. Barab, M. Thomas, T. Dodge, R. Carteaux, and H. Tuzun, "Making learning fun: Quest Atlantis, a game without guns," Educational Technology Research and Development, vol. 53, pp. 86107, 2005http://dx.doi.org/10.1007/BF02504859

[16] C. Haskell, "Understanding quest-based learning : Creating effective classroom experiences through game-based mechanics and community", Boise, USA: Boise State University, Mar 2013. 
PAPER

MERGING REMOTE LABORATORIES AND ENQUIRY-BASED LEARNING FOR STEM EDUCATION

[17] G. Siemens, "Connectivism: A learning theory for the digital age", 2004, Retrieved from elearnspace website: http://www.elearnspace.org/Articles/connectivism.htm

[18] O. A. Herrera and D. A. Fuller, "Collaborative model for remote experimentation laboratories used by non-hierarchical distributed groups of engineering students," Australasian Journal of Educational Technology, vol. 27, no. 3, pp. 428-445, 2011.

[19] T. Luis de la, "Providing Collaborative Support to Virtual and Remote Laboratories," IEEE Transactions on Learning Technologies, vol. 99, pp. 1-1, 06/04 2013.

[20] L. Divine and R. Williams, "STEM collaboration in virtual world academy," in Collaboration Technologies and Systems (CTS), 2013 International Conference on, 2013, pp. 569-575.

[21] J. Machotka, Z. Nedic, and O. Gol, "Collaborative learning in the remote laboratory NetLab," Imsci '07: International MultiConference on Society, Cybernetics and Informatics, Vol 2, Proceedings, pp. 73-78, 2007.

[22] B. Scheucher, P. H. Bailey, C. Gütl, and J. V. Harward, "Collaborative Virtual 3D Environment for Internet-Accessible Physics Experiments," International Journal of Online Engineering (iJOE), vol. 5, 2009.

[23] D. Gillet, A. V. N. Ngoc, and Y. Rekik, "Collaborative web-based experimentation in flexible engineering education," Ieee Transactions on Education, vol. 48, pp. 696-704, Nov 2005. http://dx.doi.org/10.1109/TE.2005.852592

[24] M. A. Bochicchio and A. Longo, "Extending LMS with Collaborative Remote Lab Features," in Advanced Learning Technologies (ICALT), 2010 IEEE 10th International Conference on, 2010, pp. 310-314.

[25] M. Tawfik, C. S., D. Gillet, D. Lowe, H. Saliah-Hassane, E. Sancristobal, and M. Castro, "Laboratory as a Service (LaaS): a
Model for Developing and Implementing Remote Laboratories as Modular Components", 2014 11th International Conference on Remote Engineering and Virtual Instrumentation (REV), 26-28 February 2014, Porto, Portugal, pp.11-20.

[26] A. A. Kist, A Maxwell, P Gibbings, R Fogarty, W Midgley and K Noble, "Engineering for primary school children: Learning with robots in a remote access laboratory", WEE2011, Sept. 27-30, 2011, Lisbon, Portugal, pp. 586-591.

\section{AUTHORS}

A. Maiti is with the Faculty of Health, Engineering and Sciences, University of Southern Queensland, Toowoomba, Australia. (anandamaiti@live.com).

A. D. Maxwell is with the Faculty of Health, Engineering and Sciences, University of Southern Queensland, Toowoomba, Australia (andrew.maxwell@usq.edu.au).

Alexander A. Kist is with the Faculty of Health, Engineering and Sciences, University of Southern Queensland, Toowoomba, Australia (kist@ieee.org).

Lindy Orwin is with the Australian Digital Futures Institute, University of Southern Queensland, Toowoomba, Australia (lindy.orwin@usq.edu.au).

This work is supported through the Australian Government's Collaborative Research Networks (CRN) - Digital Futures program. It is an extended and modified version of a paper presented at the EDUCON2014 conference held at the Military Museum and Cultural Center, Harbiye, Istanbul, Turkey, 3-5 April 2014. Submitted, July, 2, 2014. Published as resubmitted by the authors on October, 25, 2014. 